\title{
The FAMU experiment: muonic hydrogen high precision spectroscopy studies
}

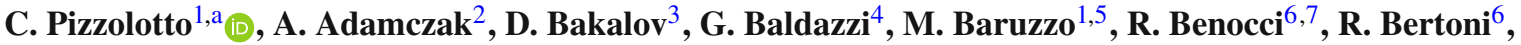 \\ M. Bonesini ${ }^{6,8}$, V. Bonvicini ${ }^{1}$, H. Cabrera ${ }^{1}$, D. Cirrincione ${ }^{1,5}$, M. Citossi $^{5}$, F. Chignoli ${ }^{6}$, M. Clemenza $^{6,8}$, L. Colace $^{9,10}$, \\ M. Danailov ${ }^{1,11}$, P. Danev ${ }^{3}$, A.de Bari ${ }^{12,13}$, C. De Vecchi ${ }^{14}$, M. de Vincenzi ${ }^{9,14}$, E. Fasci ${ }^{15}$, E. Furlanetto ${ }^{1,5}$, \\ F. Fuschino ${ }^{4,16}$, K. S. Gadedjisso-Tossou ${ }^{1,17,18}$, L. Gianfrani ${ }^{15}$, D. Guffanti ${ }^{1,19}$, A. D. Hillier ${ }^{20}$, K. Ishida $^{21}$,

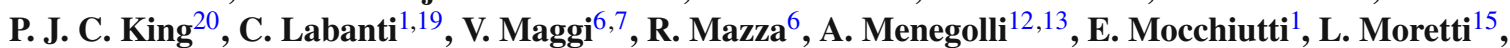 \\ G. Morgante $^{4,16}$, J. Niemela ${ }^{17}$, B. Patrizi ${ }^{22}$, A. Pirri ${ }^{23}$, A. Pullia ${ }^{24,25}$, R. Ramponi ${ }^{24,26}$, L. P. Rignanese ${ }^{4}$, \\ H. E. Roman $^{8}$, M. Rossella ${ }^{13}$, R. Sarkar ${ }^{27}$, A. Sbrizzi ${ }^{4}$, M. Stoilov ${ }^{3}$, L. Stoychev ${ }^{1}$, J. J. Suárez-Vargas ${ }^{1,5,17}$, G. Toci ${ }^{22}$,

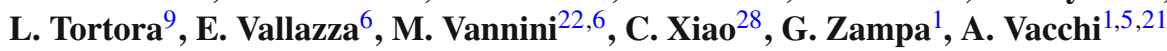 \\ ${ }^{1}$ Sezione INFN di Trieste, via A. Valerio 2, Trieste, Italy \\ 2 Institute of Nuclear Physics, Polish Academy of Sciences, Radzikowskiego 152, 31342 Kraków, Poland \\ ${ }^{3}$ Institute for Nuclear Research and Nuclear Energy, Bulgarian Academy of Sciences, blvd. Tsarigradsko ch. 72, 1784 Sofia, Bulgaria \\ ${ }^{4}$ Sezione INFN di Bologna, viale Berti Pichat 6/2, Bologna, Italy \\ ${ }^{5}$ Dipartimento di Scienze Matematiche, Informatiche e Fisiche, Università di Udine, via delle Scienze 206, Udine, Italy \\ ${ }^{6}$ Sezione INFN di Milano Bicocca, Piazza della Scienza 3, Milan, Italy \\ ${ }^{7}$ Dipartimento di Scienze dell'Ambiente e della Terra, Università di Milano Bicocca, Piazza della Scienza 1, Milan, Italy \\ ${ }^{8}$ Dipartimento di Fisica G. Occhialini, Università di Milano Bicocca, Piazza della Scienza 3, Milan, Italy \\ ${ }^{9}$ Sezione INFN di Roma Tre, Via della Vasca Navale 84, Rome, Italy \\ 10 Dipartimento di Ingegneria, Università degli Studi Roma Tre, Via V. Volterra 62, Rome, Italy \\ ${ }^{11}$ Sincrotrone Elettra Trieste, SS14, km 163.5, Basovizza, Italy \\ 12 Dipartimento di Fisica, Università di Pavia, via A. Bassi 6, Pavia, Italy \\ 13 Sezione INFN di Pavia, Via A. Bassi 6, Pavia, Italy \\ ${ }^{14}$ Dipartimento di Matematica e Fisica, Università di Roma Tre, Via della Vasca Navale 84, Rome, Italy \\ 15 Sezione INFN di Napoli e Dipartimento di Matematica e Fisica, Università della Campania "Luigi Vanvitelli”, Viale Lincoln 5, Caserta, Italy \\ 16 INAF-OAS Bologna, via P. Gobetti 93/3, Bologna, Italy \\ 17 The Abdus Salam International Centre for Theoretical Physics, Strada Costiera 11, Trieste, Italy \\ ${ }^{18}$ Laboratoire de Physique des Composants à Semi-conducteurs (LPCS), Départment de physique, Université de Lomé, Lomé, Togo \\ ${ }^{19}$ Gran Sasso Science Institute, via F. Crispi 7, L'Aquila, Italy \\ ${ }^{20}$ ISIS Neutron and Muon Source, STFC Rutherford-Appleton Laboratory, Didcot OX11 0QX,, UK \\ ${ }^{21}$ Riken Nishina Center, RIKEN, 2-1 Hirosawa, Wako, Saitama 351-0198, Japan \\ 22 INO-CNR, via Madonna del Piano 10, 50019 Sesto Fiorentino, Italy \\ ${ }^{23}$ IFAC-CNR, via Madonna del Piano 10, 50019 Sesto Fiorentino, Italy \\ ${ }^{24}$ Sezione INFN di Milano, via Celoria 16, Milan, Italy \\ 25 Dipartimento di Fisica, Università degli Studi di Milano, via Celoria 16, Milan, Italy \\ ${ }^{26}$ IFN-CNR, Dipartimento di Fisica, Politecnico di Milano, piazza Leonardo da Vinci 32, Milan, Italy \\ ${ }^{27}$ Indian Centre for Space Physics, Kolkata, India \\ ${ }^{28}$ Dalian Institute of Chemical Physics of the Chinese Academy of Sciences, 457 Zhongshan Road, Dalian 116023, People's Republic of China
}

Received: 21 January 2020 / Accepted: 7 July 2020 / Published online: 20 July 2020

(C) The Author(s) 2020

Communicated by Klaus Blaum

\begin{abstract}
The FAMU experiment aims to measure for the first time the hyperfine splitting of the muonic hydrogen ground state. From this measurement the proton Zemach radius can be derived and this will shed light on the determination of the proton charge radius. In this paper, we describe the scientific goal, the method and the detailed preparatory
\end{abstract}

work. This includes the outcome of preliminary measurements, subsequent refined simulations and the evaluation of the expected results. The experimental setup being built for the measurement of the hyperfine splitting to be performed at the RAL laboratory muon facility is also described.

a e-mail: cecilia.pizzolotto@ts.infn.it (corresponding author) 


\section{Introduction}

Muonic hydrogen allows high-precision spectroscopy studies of the fundamental interactions of the proton and its structure. Compared to ordinary hydrogen, the typical binding energies and distances in the muonic hydrogen atom are rescaled by the ratio of their reduced masses $f=m_{p \mu} / m_{p e}=$ 185.83. This directly impacts the following observable physical phenomena:

1. the radius of the muon orbit in the ground state of muonic hydrogen is of the order of $a_{0} / f=285 \mathrm{fm}$, where $a_{0}$ is the Bohr radius; this is far below the electron orbiting distances in ordinary hydrogen. The relativistic and higher order QED effects on the energy spectrum are enhanced as powers of the factor $f$; in particular, the hyperfine splitting is enhanced as $f^{2}$;

2. the overlap of muon and proton densities is enhanced by $f^{3}$ : thus the effects of the spatial distribution of the proton charge and magnetic moment becomes accessible;

3 . the re-scaled transition energies between levels in muonic hydrogen are shifted to spectral ranges which allow for high precision laser spectroscopy;

4. the nature of this very sensitive atomic system and the constant progression in the theoretical calculations offers a unique opportunity to experimentally explore the fundamental interactions at low momentum-transfer [1-4].

To measure the hyperfine splitting $(h f s)$ in the ground state of muonic hydrogen $\Delta E^{h f s}\left(\mu^{-} p\right)_{1 S}$ with a relative accuracy better than $10^{-5}$, an intense, pulsed, low-energy, negative muon beam and an appropriate mid-infrared laser source are required [5-7]. The measurement will contribute to the following key challenging aspects of the problem:

- determination with at least $1 \%$ accuracy, of the Zemach radius of the proton $r_{Z}$, the first moment of the convolution of its electric charge and magnetic moment distributions;

- resolution of the ambiguities about the electromagnetic structure of proton through completion of a cycle of highprecision exotic atom counterparts of the historical measurements of the Lamb shift and the hyperfine splitting in ordinary hydrogen, and providing a step of great importance as a fundamental test of QED [8-10]. The current ambiguity creates difficulty for the determination of the Rydberg constant [11];

- the exceptional precision of $10^{-12}$ of the experimental value of $\Delta E^{h f s}$ in hydrogen [9] makes it sensitive, in addition to QED corrections, to the contribution from the proton finite size and proton polarizability. They cannot be extracted at the same time from a single experiment. A measurement of $\Delta E^{h f s}\left(\mu^{-} p\right)_{1 S}$ with an accuracy better than $10^{-5}$ provides another independent combination of the non-QED part and will be a significant step forward in the study of the proton structure. In fact, in natural hydrogen the knowledge of the proton size is the limiting factor in the comparison of the measured hyperfine structure with theory. The poor knowledge of proton parameters presently limits any comparison between theory and the measured values;

- by testing the limits of the most precise theoretical predictions, it is possible to become sensitive to new phenomena and potentially probe physics beyond the Standard Model and connect the fields of atomic and high energy physics.

The Zemach radius $r_{Z}$ and the r.m.s. charge radius $r_{c h}$ are the only values, related to the proton shape, that can be directly extracted from experimental spectroscopic data. The Zemach radius is the only one that carries information about the proton's magnetic dipole moment distribution. From recent theoretical predictions $[1,12-15]$ one has:

$$
\begin{aligned}
& \Delta E^{h f s}\left(\mu^{-} p\right)_{1 S}=182.819(1)[\mathrm{meV}] \\
& \quad-1.301[\mathrm{meV} / \mathrm{fm}] r_{Z}[\mathrm{fm}]+0.064(21)[\mathrm{meV}] .
\end{aligned}
$$

The first term includes the Fermi energy, QED corrections, hadronic vacuum polarization, recoil corrections and weak interactions. The second term, proportional to $r_{Z}$, is the finite size contribution containing also some higher order mixed radiative finite size corrections and the third term is given by the proton polarizability contribution. Each of these terms is subject to constantly improved calculations: their specific numerical values and their uncertainties are thus subject to variations.

FAMU (Fisica degli Atomi Muonici) aims to measure $\Delta E^{h f s}\left(\mu^{-} p\right)_{1 S}$ and to extract the Zemach radius $r_{Z}$ with a relative accuracy better of $1 \%$. Up to now, $r_{Z}$ has been extracted from the $h f s$ in ordinary hydrogen by four independent groups with differing results [14-17]. A measurement of $r_{Z}$ in muonic hydrogen will be a precious contribution to the works on the proton radius measurements that have animated a debate in recent years. Published data from [18] on muonic deuterium are compatible with the previously reported muonic hydrogen results $[19,20]$. None of them includes a direct measurement of the hyperfine splitting energy. Proposals to measure the hyperfine splitting with pure hydrogen target have been approved at PSI and JPARC $[21,22]$. This situation confirms the fundamental interest in a new and independent high precision measurement on muonic hydrogen.

The very first version of the FAMU experimental method was proposed in 1993. Only recent developments in the field of laser science and a revision of the original project 
made the measurement attainable. Measurements performed by FAMU until now were crucial to prove the feasibility of the method, but did not include a laser-cavity system. At the same time, a suitable laser system has been developed and now reached the required characteristics.

The newly developed instrumentation and full layout and integration of the experiment is presented here for the first time. In Sect. 2 the experimental method of the FAMU experiment is described, including the preparatory work that proved the feasibility of the measurement. Section 3 describes the entire apparatus, including the laser system, the optical cavity, the gaseous target, and X-rays and beam detectors. Finally, in Sect. 4 we discuss the foreseen measurement plan.

\section{The FAMU experiment}

\subsection{The muon transfer method}

The progression leading to the proposed method in FAMU for the measurement of the magnetic M1 transition in $\left(\mu^{-} \mathrm{p}\right)_{1 S}$ between the hyperfine $\mathrm{F}=0$ and $\mathrm{F}=1$ states can be found in Refs. [5-7,23-28]. The sequence of physical processes behind this method is shown schematically in Fig. 1. Muonic hydrogen is formed, thermalizes, depolarizes and propagates in a gas target containing a mixture of hydrogen and oxygen. At the right time after the formation, a thermal muonic hydrogen atom in the para state (total spin $\mathrm{F}=0$ ) exposed to photons at the resonance energy $\Delta E^{h f s} \simeq 0.182 \mathrm{eV}(6.8 \mu \mathrm{m})$ can be excited to the ortho $(\mathrm{F}=1)$ spin state. Very quickly, in subsequent collisions with the surrounding $\mathrm{H}_{2}$ molecules, muonic hydrogen de-excites to $(\mathrm{F}=0)$ [29]. Because of energy and momentum conservation, at the exit of the de-excitation collision, the muonic hydrogen is accelerated by $0.1 \mathrm{eV}$ which is $\sim 2 / 3$ of the $\Delta E_{h f s}$ excitation energy. The muon is transferred from $\mu^{-} \mathrm{p}$ to form $\mu^{-} \mathrm{O}$ at a rate $\lambda_{O}(\mathrm{E})$ that increases considerably with the energy of the $\mu^{-} \mathrm{p}$, in the energy interval up to about $0.2 \mathrm{eV}[30,31]$. The de-excitation of muonic oxygen produces characteristic X-rays: $\mathrm{K}_{\alpha} \simeq 133 \mathrm{keV}, \mathrm{K}_{\beta} \simeq$ $158 \mathrm{keV}, \mathrm{K}_{\gamma} \simeq 167 \mathrm{keV}$. By varying the wavelength of the tunable laser, it is possible to experimentally identify the resonance wavelength as the value for which the number of spin-excited atoms and hence of the X-rays from transfer to oxygen is maximised.

Summarizing, the sequence of physical processes will be the following:

$-\mu^{-} \mathrm{p}$ formation and the thermalization phase: in the high purity mixture of hydrogen and oxygen, at appropriate temperature and pressure, muonic hydrogen atoms $\mu^{-} \mathrm{p}$ are formed, thermalized and depolarized in subsequent collisions. A fraction of the muons is progressively transferred from $\mu^{-} \mathrm{p}$ to excited states of $\mu^{-} \mathrm{O}$. Those events are recognizable by the characteristic X-rays emitted during muonic oxygen de-excitation.

- Spin-flip phase: at an appropriate time after thermalization, a laser pulse at the resonance wavelength is sent to a multipass optical cavity enclosing the gas target. If the spin-flip transition occurs, the time distribution of the characteristic X-rays from muon transfer to oxygen will be perturbed.

- The hyperfine splitting resonance wavelength value $\lambda h f s$ is recognized by a maximal difference between the time distribution of the muon transfer events to oxygen with and without a laser pulse.

In Fig. 2 measurements taken with $\mathrm{LaBr}_{3}(\mathrm{Ce})$ detectors reveal the progressive evolution of the prompt (immediately after the arrival of the beam pulses) and delayed oxygen muonic X-ray lines. The prompt muonic X-ray lines from aluminium and the other target's components during the muon beam spill is shown in panels (a) and (c); subsequently, the

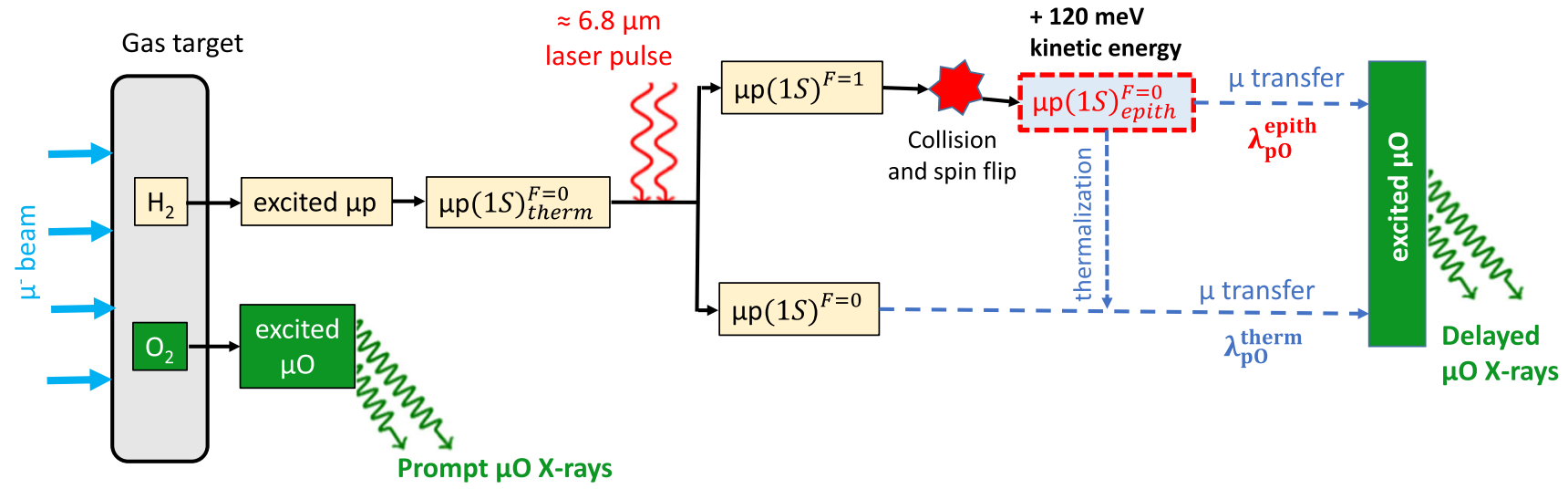

Fig. 1 Schematic representation of the FAMU experimental method. In this left-to-right time progression: muons stop in the gas target and subsequently de-excite and thermalize. At a latter time, the laser excita- tion prompts the formation of the $\mathrm{F}=1$ state. This will cause a sizeable change in the time distribution of the delayed X-rays coming from muon transfer to oxygen 

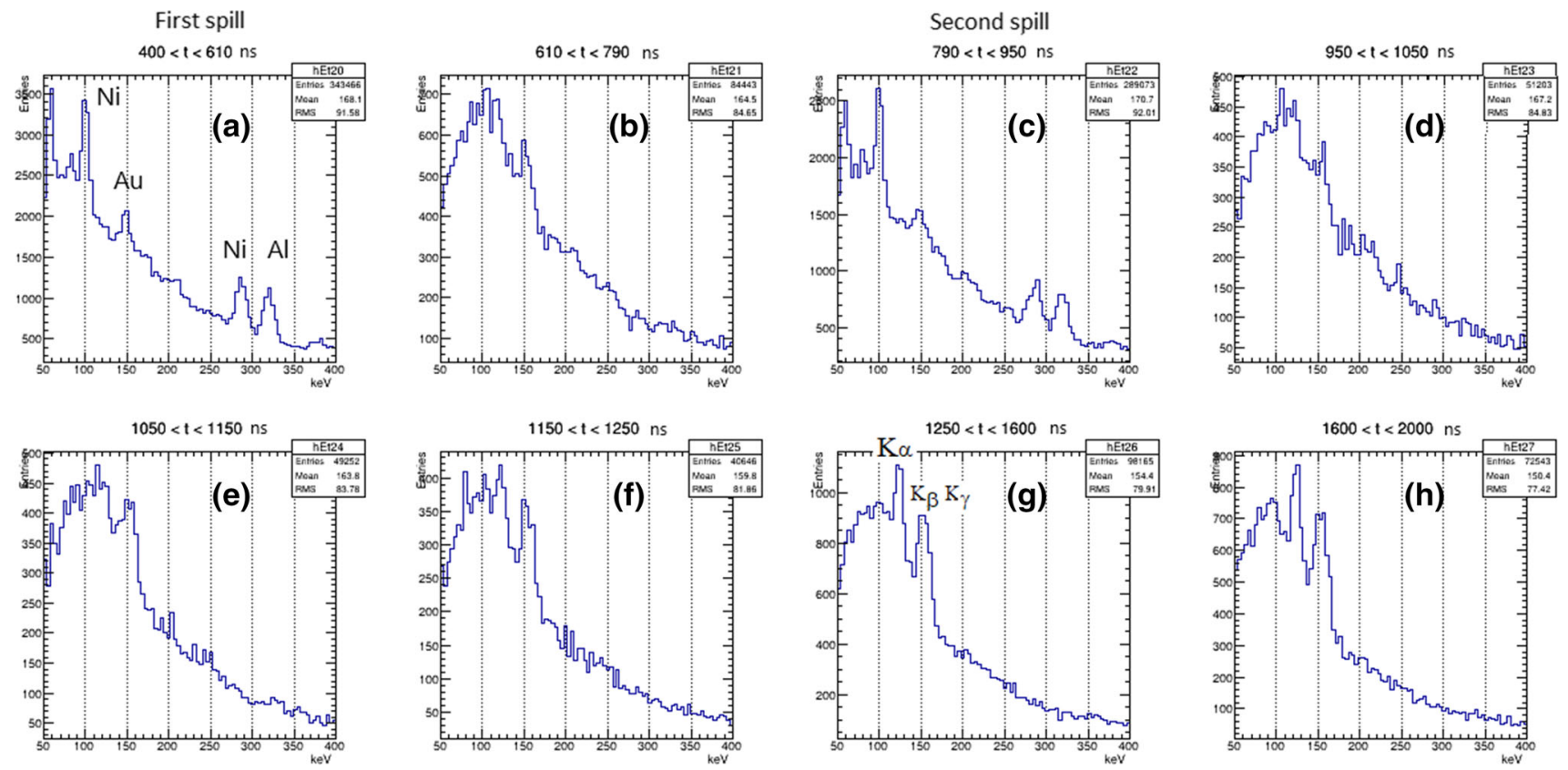

Fig. 2 The evolution of the energy spectra registered by one $\mathrm{LaBr}_{3}(\mathrm{Ce})$ detector (real data) at different times during and after the arrival of the double pulsed muon beam spill (see Sect. 3.1)

oxygen lines from delayed $\mu^{-} \mathrm{p}$ transfer to oxygen appear, panels (f), (g), and (h). We plan to inject the laser beam as soon as the muonic hydrogen is thermalized. This time slot corresponds to panel (g) of Fig. 2; as can be seen the delayed oxygen X-rays lines are already well defined over the background. The time evolution of the oxygen muonic K-lines is determined by the gas parameters: pressure and oxygen concentration. By properly adjusting these parameters, we can set the best conditions for the laser injection, as discussed in Sect. 2.3.1.

\subsection{From hyperfine splitting in muonic hydrogen to the determination of the proton Zemach radius}

The probability $P$ for the laser radiation to stimulate a hyperfine transition is expressed in terms of the energy $E$ of the laser pulse (in J), the cross section of the illuminated cavity $S$ (in $\mathrm{cm}^{2}$ ), the target temperature $T$ (in $\mathrm{K}$ ) and the reflectivity $R$ of the multipass cavity mirrors (under the assumption that the laser linewidth is smaller than the transition linewidth) as follows [6]:

$P=2 \cdot 10^{-5} \cdot \frac{E}{(1-R) \cdot S \cdot \sqrt{T}}$.

The probability $P$ will reach $\sim 45 \%$ at the final stage of the FAMU experimental setup, with the parameters $E=4 \mathrm{~mJ}$; $T=80 \mathrm{~K} ; S=1 \mathrm{~cm}^{2} ; R=0.9998$.

The main source of uncertainty in the experimental value of the resonance frequency $v_{h f s}=\Delta E_{h f s} / h$ is the statistical uncertainty. The estimate [6] of the relative statistical uncertainty on $v_{h f s}$ is:

$$
\frac{\delta v_{h f s}}{v_{h f s}}=0.866 \cdot \frac{1}{\sqrt{m}} \cdot \frac{1}{\rho} \cdot \frac{\Gamma}{v_{h f s}},
$$

where $\rho$ is the signal-to-noise ratio

$\rho=\frac{\left(N_{L}-N_{0}\right)}{\sqrt{2\left(N_{L}+N_{0}\right)}}$.

$N_{L}$ and $N_{0}$ are the number of muon transfer events in the time window with and without a laser shot, $\Gamma$ is the width of the scanned interval of frequencies and $m$ is the number of wavelengths measurements in the scanned interval. The measurement of the resonance hyperfine transition frequency $v_{h f_{S}}=\Delta E^{h f_{s}} / h$ with an accuracy $\delta v_{h f_{S}} / \nu_{h f_{S}} \sim 10^{-5}$ is a realistic goal of the project, considering $m \simeq 100, \Gamma=$ $20 \mathrm{GHz}$, and $\rho$ at least four.

The determination of the Zemach radius from the experimental value of the hyperfine splitting is based on the theoretical relation between the hyperfine splitting $\Delta E^{h f s}$, the lowest order Fermi hyperfine energy $E_{F}$ and the corrections to it $\delta^{Q E D}$ due to QED effects, $\delta^{r e c}$ recoil, $\delta^{Z}$ the static electromagnetic structure of the proton, $\delta^{p o l}$ to dynamical proton polarizability and $\delta^{h v p}$ to hadron vacuum polarization respectively. The relation is written as:

$\Delta E^{h f s}=E_{F}\left(1+\delta^{Q E D}+\delta^{r e c}+\delta^{Z}+\delta^{p o l}+\delta^{h v p}\right)$.

Of these quantities $E_{F}, \delta Q E D$ and $\delta^{r e c}$ are known or calculable with accuracy $10^{-6}$ or better, and $\delta^{h v p}$ is small and may 
be neglected. The term $\delta^{Z}$ is related to the Zemach radius $r_{Z}$ by means of

$\delta^{Z}=2 \alpha(1+k) \cdot \frac{M_{\mu} M_{p}}{M_{\mu}+M_{p}} \cdot r_{Z}$,

where $M_{\mu}$ and $M_{p}$ are the particle masses, $\alpha$ the fine structure constant and $k=0.0152$ is a QED correction [32], giving approximately $\delta^{Z}=-7.3 \cdot 10^{-3}$. Using phenomenological data, the proton polarizability term $\delta^{\text {pol }}$ was evaluated to $\delta^{p o l}=(4.6 \pm 0.8) \cdot 10^{-4}[33]$. In this way, the uncertainty in the value of the Zemach radius obtained by resolving Eqs. (5) and (6) with respect to $r_{Z}$ is limited by the uncertainty of $\delta^{p o l}$ to about $1 \%$. As already pointed out, knowing $r_{Z}$ with the accuracy of $\Delta r_{Z}=0.01 \mathrm{fm}$ will be a sufficiently strong result to confirm or reject with high confidence the existence of any peculiarities in the $\mu^{-} \mathrm{p}$ interaction.

To date, published values of the Zemach radius are (in $\mathrm{fm}$ ): 1.037(16), 1.043(16), 1.045(16), 1.086(12) from [14-17] and more recently 1.082(37) [20] and 1.045(4) [34]. FAMU measurement will improve by about a factor two the experimental data accuracy. In the meantime, the experimental results will stimulate improved accuracy calculations of $\delta^{p o l}$, that will in turn allow to extract the proton Zemach radius with a still higher precision.

\subsection{Preparatory work}

The FAMU experiment takes place at the RIKEN-RAL muon beam facility at the Rutherford-Appleton Laboratory, in the Oxfordshire (UK) [35]. Since 2013, the FAMU team had four very fruitful data taking sessions at the facility demonstrating that the proposed method, the muon beam characteristics and the chosen detection systems are all suitable for the proposed task $[36,37]$. The setup did not include the laser-cavity system. FAMU published a number of technical and scientific results $[7,36-42]$. From these data taking periods, we were able to:

- confirm the suitability of the transfer method for oxygen [7,37];

- investigate different gas configurations to determine the best final setting (from the type and quantity of high $\mathrm{Z}$ element in gas mixture, to values of pressure and temperature);

- estimate the minimal duration of a run in the final configuration.

The last item is presented in Sect. 4.

\subsubsection{Feasibility of the transfer method for oxygen}

The efficiency of the proposed method relies on the muon transfer rate dependence on the muonic hydrogen energy and on the epi-thermality of the muonic hydrogen at the moment of the muon transfer. While for many admixed gases the transfer rate of the muon from hydrogen at low energies is nearly constant, there is experimental evidence, confirmed by the recent experimental study performed by FAMU, that the transfer to oxygen has a marked energy dependence $[30,37,43]$. In 2016, in the first investigation of the temperature dependence of the muon-transfer process from the thermalized $\mu^{-} \mathrm{p}$ atoms to oxygen, a strong monotonic rise of the transfer rate to oxygen in the temperature interval 104-300 K has been observed [38]. Based on this data, the energy dependence of the transfer rate has been quantitatively determined: it increases by a factor of about eight in the collision-energy interval $[0.01,0.08] \mathrm{eV}$. Such a strong change enables us to employ the muon transfer rate to oxygen as a signature of the kinetic-energy gain of the $\mu^{-} \mathrm{p}$ atom. This result not only sets constraints on theoretical models of muon transfer, but it is also of fundamental importance for the measurement of the hyperfine splitting of $\mu^{-} \mathrm{p}$. With this experiment we have reached a confirmation that oxygen is an appropriate gas for the transfer rate method.

\subsubsection{Monte Carlo simulation of the physical processes}

A detailed simulation of the physical processes is required for an accurate determination of the signal to noise ratio as a function of the gas pressure and oxygen concentration that will allow to reach optimal conditions for the experiment. The thermalization of the accelerated $\mu^{-} \mathrm{p}$ has to be slower than the epithermal $\mu^{-}$transfer, this dictates the physical conditions of the gas mixture in the target. The $\mu^{-} \mathrm{p}$ scattering and muon transfer process and the role of these processes in the measurement of the hyperfine splitting $\left(\mu^{-} \mathrm{p}\right)_{1 S}$, have been subject of detailed investigations $[28,43,44]$.

At higher pressures, deceleration (thermalization) of $\mu^{-} \mathrm{p}$ atoms is fast compared to the muon-transfer process. Thus, for higher pressures, the laser light can be shot earlier when more $\mu^{-} \mathrm{p}$ atoms are present at the instant of the laser pulse. On the other hand, fast thermalization is a negative effect because it reduces the number of muon transfers from energetic $\mu^{-} p$ atoms to oxygen. High concentration of oxygen is preferred to give more $\mathrm{X}$-rays signals, but too high a concentration would consume all muons in the prompt phase, during the muon arrival. A Monte Carlo simulation has been developed by our team to optimize these aspects of the FAMU experiment. The code includes the description of the muon decay, $\mu^{-} \mathrm{p}$ scattering from the $\mathrm{H}_{2}$ molecules, formation of the $\mathrm{pp} \mu^{-} \mathrm{p}$ and of the $\mathrm{pd} \mu^{-}$molecules, muon transfer between proton and deuteron and, of course, of the muon transfer to oxygen. It also includes the energy dependence of the muon tranfer rate, that has been fitted to the FAMU [38] and PSI data [30]. This numerical simulation is used to determine the optimal gas temperature, pressure and compo- 


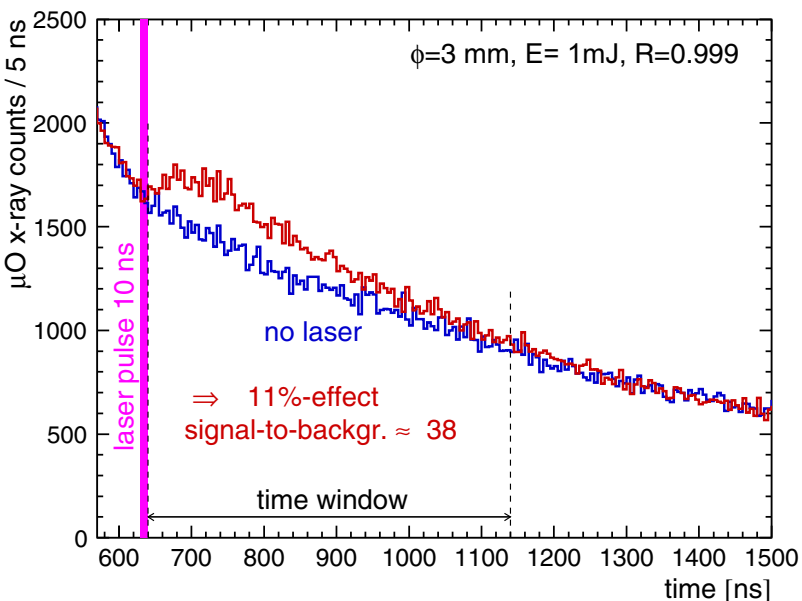

Fig. 3 Results of the Monte Carlo simulation of the muonic oxygen de-excitation X-ray time distribution. This simulation does not include a full simulation of the FAMU detector. The two panels show the effect in a volume completely filled by a laser of $3 \mathrm{~mm}$ diameter and $1 \mathrm{~mJ}$

sition, the best timing for the laser shot and the time window to be considered for the final spectroscopic stage. For the gas target conditions the optimal combination at $80 \mathrm{~K}$ was found for a pressure of about 7 bar and $1.5 \%$ (weight) oxygen concentration. The Monte Carlo simulations do not include the direct muon transfer to oxygen (and muon transfer from excited $\mu^{-}$p) within prompt peaks. Some significant simulation's results are presented in Fig. 3. The figure shows the delayed de-excitation X-ray time distribution obtained for a fixed oxygen concentration of $1 \%$ and the gas pressure of 7 bars without (blue line) and with laser (red line). The laser induces the spin flip transition and the subsequent change in the X-ray time distribution. The two panels show results at different laser energy densities. Part of this study was aimed at the determination of the optimal conditions on the concentration-pressure plane. The only geometric parameter is the mirror distance $d=10 \mathrm{~cm}$, which determines the lifetime of the laser field. Notice the effect in the real cavity will be smaller than what is depicted in Fig. 3, since a portion of the gas is not illuminated.

In 2018, FAMU acquired a set of data at different combination of pressure and oxygen concentration. These data validate experimentally the results of this simulation and confirm the definition of optimal target condition for the measurement.

\section{The FAMU experimental setup}

In the forthcoming paragraphs, we describe the main aspects and requirements of the FAMU experiment in the final setting:

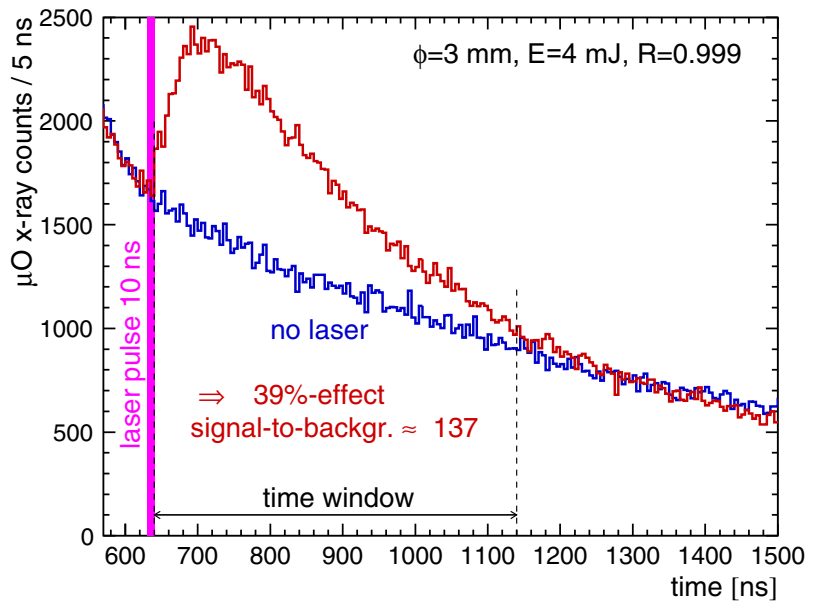

energy (left panel) or $4 \mathrm{~mJ}$ (right panel). The effect, defined as the difference between the oxygen X-rays distribution with and without laser (red and blue lines) normalized to the no-laser distribution, is of $11 \%$ in the case of $1 \mathrm{~mJ}$ and $39 \%$ in the case of $4 \mathrm{~mJ}$

- intense low momentum (30-80 MeV/c) pulsed negative muons beam;

- pulsed tunable narrow-band laser in the wavelength range of $6.8 \mu \mathrm{m}$ (mid-infrared) with energy output of approximately $4 \mathrm{~mJ}$, to stimulate the hyperfine transition;

- multipass high reflective optical cavity, to raise the excitation efficiency of the muonic hydrogen atoms formed in the gas target;

- cryogenic pressurized high purity hydrogen gas target;

- beam profile monitor;

- fast detection system for X-rays with efficiency and energy resolution optimized for the detection of the oxygen muonic X-rays which give the signature of the transition.

All elements will be placed on a single structure, an optical table of $1.5 \times 3.5 \mathrm{~m}^{2}$. Figure 4 shows a schematic representation of the setup. The laser system occupies most of the optical table and is covered by panels, for safety reasons. The cryostat with the target is held in place by an adjustable structure that permits the alignment of the cavity to the beam at the beginning of the run. The vertical pipe joined to the horizontal box encloses the laser optical path to the multipass cavity. This representation shows also the hodoscope, a partial crown of detectors around the target and a germanium detector, its nitrogen tank is recognisable at the back/left of the picture. The red cylindrical shape is the new beam collimator, the only piece in this $\mathrm{CAD}$ view that is not supported by the table. 


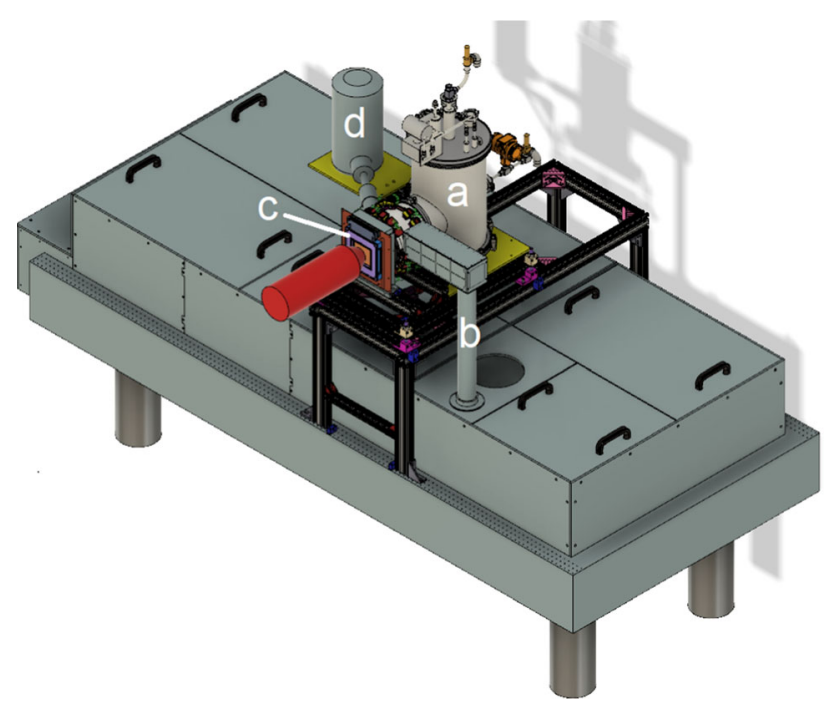

Fig. 4 CAD representation of the full FAMU system. Letters indicate some of the elements: a cryostat; b laser optical path; c hodoscope; $\mathbf{d}$ nitrogen tank for the germanium detector. Description in the text

\subsection{Muon beam}

We have chosen the RIKEN-RAL muon facility at the ISIS accelerator of the Rutherford-Appleton Laboratory [35]. Here, up to $10^{5}$ (momentum dependent) negative muons per second can be delivered. The beam momentum can be tuned in the range $30-80 \mathrm{MeV} / \mathrm{c}$, with a double pulse structure, a repetition rate of $50 \mathrm{~Hz}$, a momentum spread $\sigma_{p} / \mathrm{p} \approx 4 \%$, and a beam transverse section $\sigma_{x}, \sigma_{y}=1.5 \mathrm{~cm}$. The facility has four beam delivery ports. Port 1 is the best candidate to host the experiment, having more space for the layout, in particular for the laser system. The last beam collimator has been substituted with a new one to match to the needs of our experiment in order to maximize the number of muons in the gaseous target. The new beam collimator is shown in red in Fig. 4.

\subsection{Narrowband mid-infrared pulsed laser}

The pulsed, tunable narrow-band FAMU laser [45] is being developed to the parameters shown in Table 1. The scheme, shown in Fig. 5, is based on direct difference frequency generation (DFG) in non-oxide crystals: lithium thioindate $\left(\mathrm{LiInS}_{2}\right)$ or lithium selenoindate $\left(\mathrm{LiInSe}_{2}\right)$. It uses as pump lasers two narrow bandwidth solid state lasers: a fixed wavelength one and a tunable one, both emitting at wavelengths below $2 \mu \mathrm{m}$.

This solution is based on mixing single longitudinal mode Nd:YAG laser $(1.064 \mu \mathrm{m})$ and a tunable Cr:forsterite laser $(1.262 \mu \mathrm{m})$ pumped by a second Nd:YAG synchronized to the first one. This is an attractive scheme due to its compactness, energy scalability, and ability to fulfill the required
Table 1 Reference parameters for the pulsed FAMU laser

\begin{tabular}{lll}
\hline Wavelength range & $6800 \pm 50 \mathrm{~nm}$ & $\approx 44 \mathrm{THz}$ \\
Energy output & $>1 \mathrm{~mJ}$ & Progressiv. up to $>4 \mathrm{~mJ}$ \\
Linewidth & $<0.07 \mathrm{~nm}$ & $450 \mathrm{MHz}$ \\
Tunability steps & $0.03 \mathrm{~nm}$ & $200 \mathrm{MHz}$ \\
Pulses duration & $10 \mathrm{~ns}$ & \\
Repetition rate & $25 \mathrm{~Hz}$ & \\
\hline
\end{tabular}

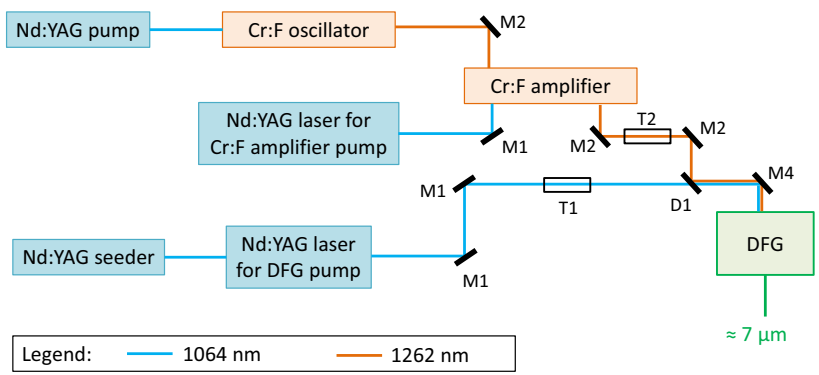

Fig. 5 Block scheme of the FAMU laser system. Letters indicate $M=$ mirror, $T=$ telescope, $D=$ dichroic mirror

laser parameters as tunability and narrow linewidth. The first results obtained by a test system, based on direct DFG emitting nanosecond pulses of infrared tunable radiation in a spectral range around $6.8 \mu \mathrm{m}$, showed that the $\mathrm{LiInS}_{2}$ is one of the most appropriate crystals for such a purpose $[45,46]$.

The test showed that, with an appropriate optimization of pumping lasers (Nd:YAG and Cr:forsterite) and by using commercially available nonlinear $\mathrm{LinS}_{2}$ and $\mathrm{LiInSe}_{2}$ crystals, it is possible to reach $1-1.5 \mathrm{~mJ}$ in the $6.8 \mu \mathrm{m}$ spectral region and a linewidth $<30 \mathrm{pm}$ [47]. Recently, a new large $\mathrm{BaGa}_{4} \mathrm{Se}_{4}$ crystal, suitable for DFG at the frequency we need, has been acquired. With this crystal, we expect to obtain an output energy exceeding $4 \mathrm{~mJ}$ at $6.8 \mu \mathrm{m}$.

For the generation of an energy greater than $1 \mathrm{~mJ}$ at $6.8 \mu \mathrm{m}$, the Nd:YAG laser must have an energy of the order of $70 \mathrm{~mJ}$ and the Cr:forsterite of $35 \mathrm{~mJ}$. At present, we have a commercially available single-frequency mode Nd:YAG laser system with output energy $>250 \mathrm{~mJ}$. We have built a multipass multi-stage amplifying system with finely tunable wavelenght and narrow bandwidth at $1.262 \mu \mathrm{m}$ able to reach $24 \mathrm{~mJ}[47,48]$. To obtain a linewidth of $250 \mathrm{MHz}$ at $6.8 \mu \mathrm{m}$, the linewidths of both pump lasers will be narrowed:

- at $1.064 \mu \mathrm{m}$ to $30 \mathrm{MHz}$ by integrating an iodine cell,

- at $1.262 \mu \mathrm{m}$ to $100 \mathrm{MHz}$ using a tunable laser seeder.

The wavelengths of the pump lasers depend on the oscillators' resonators lengths. An active temperature stabilization is being implemented to stabilize the central wavelengths of pump lasers. 


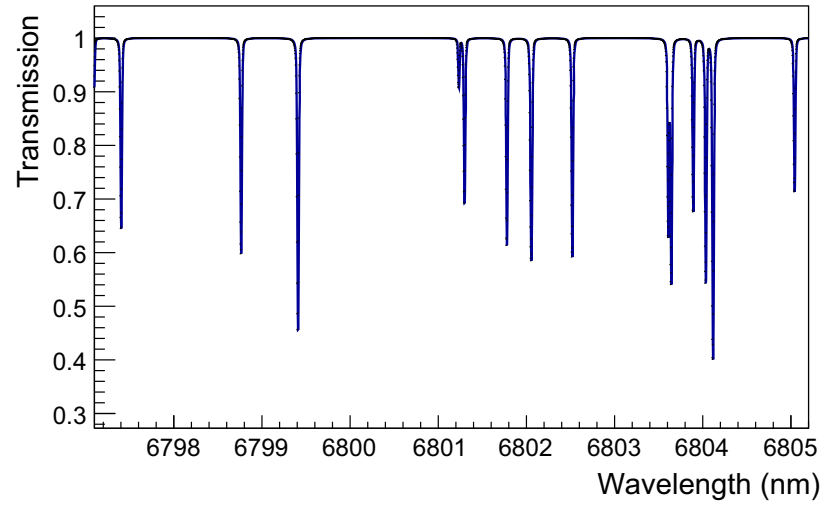

Fig. 6 Absorption spectra of ${ }^{12} \mathrm{C}_{2} \mathrm{H}_{4}$ in a range of interest, temperature $296 \mathrm{~K}$; concentration $100 \%$; pressure 5 mbar; absorption length $10 \mathrm{~cm}$. Data from [49]

At present, wavelength measurement of the DFG emission at $6.8 \mu \mathrm{m}$ is performed by a wavelength meter, based on solid state Fizeau interferometers (WS6-200 IR III, HighFinesseANGSTROM), that gives the possibility to measure both the central wavelength and the linewidth with absolute accuracies of $200 \mathrm{MHz}$ and $400 \mathrm{MHz}$ respectively, in the spectral range $2-11 \mu \mathrm{m}$. In order to provide a cross check, we are planning to acquire a wavelength meter covering the spectral range $0.532-1.750 \mu \mathrm{m}$ with absolute accuracy:

$-0.530-1.100 \mu \mathrm{m}: \pm 60 \mathrm{MHz}$

$-1.100-1.750 \mu \mathrm{m}: \pm 40 \mathrm{MHz}$,

corresponding to $0.22 \mathrm{pm}$ at $1.064 \mu \mathrm{m}$ and $0.21 \mathrm{pm}$ at $1.262 \mu \mathrm{m}$. This will provide the exact values of the wavelengths of the two pump lasers with an accuracy limited by the emitted linewidth, corresponding to an uncertainty in the estimation of the wavelength at $6.8 \mu \mathrm{m}$ of less than $30 \mathrm{pm}$.

The wavelength meter device gives the value of the measured wavelength for every pulse, so in case of unexpected change in the emission of one of the lasers and hence in the DFG emission, we will be able to control the exact wavelength. Nevertheless, an absolute calibration of the laser system is needed. An absorption cell will grant an absolute calibration of the laser tuning and control system. $\mathrm{A} \mathrm{C}_{2} \mathrm{H}_{4}$ cell has been chosen: it has absorption lines well suited for this task, as shown in the absorption spectrum in Fig. 6. The central frequency of the absorption lines shown in Fig. 6 is reported in spectral databases such as HITRAN [49]. The accuracy ranges from \pm 10 to $\pm 140 \mathrm{MHz}$, at $3 \sigma$, depending on the intensity (stronger lines are more accurate).

A laser control system has been realized to fulfill several tasks, among them: the remote control of the laser, the transfer of laser parameters to the acquisition system during data taking, the automatic emergency shutdown control.

\subsection{Target system}

The temperature of the gas shall be the lowest possible to maximize the transition probability $P$, according to Eq. 2 . At the same time, since we work with a gas mixture of hydrogen and oxygen, the condensation temperature of oxygen limits the lowest possible temperature to about $60 \mathrm{~K}$. Further requirements that should be met by the target system are:

- the beam entrance window must be thin enough to minimize the muon stop and to minimize the spread of the muon beam due to the Coulomb scattering;

- low-Z materials should be used in order to improve the transparency to the X-rays of the muonic lines of interest (50-300 keV);

- high-Z materials should be used to permit a fast nuclear capture of the muons stopped outside the gas in order to minimize the noise caused by the electrons coming from the decay of muons;

- the system must operate in safe conditions with ultrapure pressurized hydrogen gas down to liquid nitrogen temperature;

- the system must host the highly reflective optical cavity and to allow the inclusion of an optical high transparency window in the mid infrared.

A delicate balance and compromise must be found in order to comply with all these competing constraints. A study of the new target design has been carried ahead in collaboration with Criotec Impianti s.r.l. ${ }^{1}$ A dedicated Monte Carlo simulation allowed us to optimize the shape and materials of the whole target system. This simulation is described in Sect. 3.3.3.

\subsubsection{Cryogenic system}

The optical path leading the laser light towards the internal optical cavity is sensitive to vibrations. For this reason, cooling is based on a liquid nitrogen system. This optimizes mechanical stability and eliminates the vibrations produced by cryogenic pumps. The new design is shaped on the needs of the optical cavity that it hosts. The current design also allows the implementation of an improved cavity in the near future, in case a more powerful laser will be available. Figure 7 shows the drawing of the actual target design study, which has led to the choice of a liquid nitrogen $\left(\mathrm{LN}_{2}\right)$ tank of 5 liters. This, with the foreseen load of about $1.7 \mathrm{~W}$, will allow a duty cycle of about 5 days at the operating temperature of $80 \mathrm{~K}$. The $\mathrm{LN}_{2}$ refilling system will have a feeding and purging pipes always in place and will be connected to a $100 / 500$ liter dewar. By pumping on the $\mathrm{LN}_{2}$ bath one could

\footnotetext{
${ }^{1}$ https://www.criotec.com.
} 


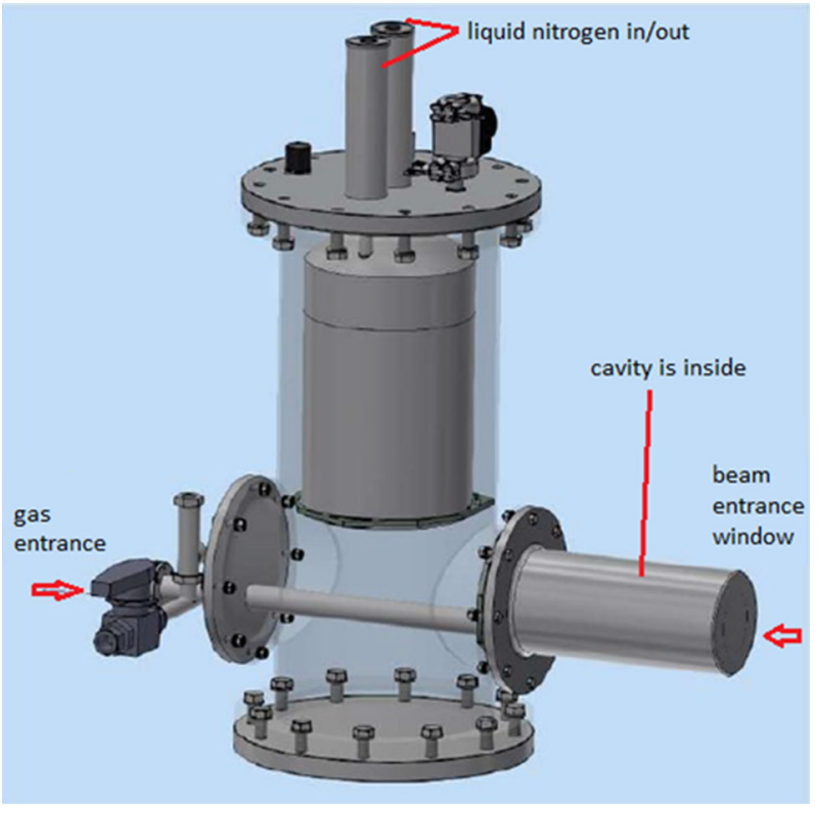

Fig. 7 The present design study of the cryogenic gas target system: view on the internal element distribution. To the right one can see the beam entrance window. The optical cavity is not visible and it is placed just behind the beam entrance window. In the vertical vessel the inlet and outlet to the liquid nitrogen tank, providing $80 \mathrm{~K}$ temperature stabilization, are visible

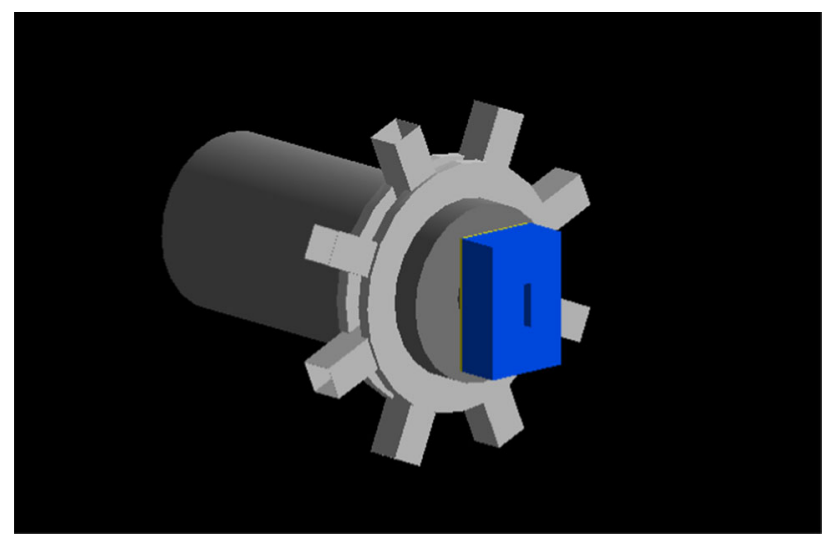

Fig. 8 GEANT4 drawing of the lead collimator (in blue), of the external cryogenic vessel, and of a star-design detector holder

establish to work at even lower temperature (down to $65 \mathrm{~K}$ ): this alternative is being considered for future development. Two temperature sensors together with a resistance heater and a digital pressure sensor will allow remote control. A $\mathrm{LN}_{2}$ level sensor is placed inside the tank.

Figure 8 shows the drawing of the target implemented in the GEANT4 simulation.

Muons traverse the lead collimator opening and enter the cryogenic cylinder through an aluminated mylar window $0.2 \mathrm{~mm}$ thick.

\subsubsection{The pressurized gas target system}

The key point in the design of the target system is the maximization of the number of muons stopping in the small amount of gas contained in the optical cavity while keeping as low as possible the noise coming from muons stopping elsewhere.

The design has been progressively refined by means of simulations reproducing all the materials present in the target, including the optical cavity structure and mirrors, described in the next section.

The illuminated volume inside the optical cavity will have dimensions of about $2 \times 2 \times 10 \mathrm{~cm}^{3}$. The $10 \mathrm{~cm}$ distance between the mirrors permit a light containment inside the cavity with no lateral mirrors. Hence, it is possible to minimize the material inside the target.

Figure 9 shows the pressurized gas target system.

The target vessel is an aluminium box, its long axis perpendicular to the cryogenic cylinder axis. The vessel has rounded corners on the front side and a removable cap on the rear. The inner walls of the pressurized cylinder are coated with gold and nickel to capture muons reaching the walls. The vessel shape was chosen to withstand the pressure with the minimum thickness of the walls. The vessel is gas tight and must permit an efficient cleaning and refilling of the gas mixture.

The mirrors are supported by a C-shaped structure made of steel connected to a main steel support plate. A thin layer $(0.6 \mathrm{~mm})$ of silver, placed inside the pressurize vessel and in front of the cavity, slows down muons just before entering the illuminated region, both increasing the muon stop and suppressing the noise coming from the aluminium vessel.

The rear support is covered with $3 \mathrm{~mm}$ thick lead which acts as a "beam stopper", absorbing most of the muons that enter the cylinder and do not stop in the gas. The rear cap is screwed to the vessel body and sealing is achieved using a malleable indium O-ring.
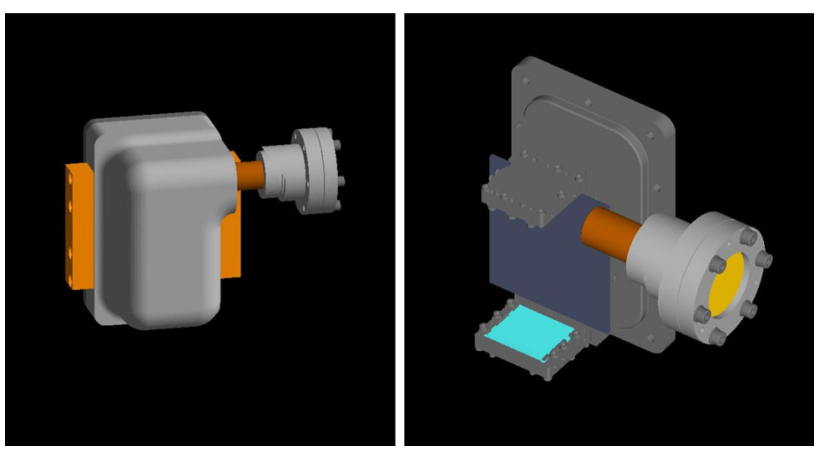

Fig. 9 GEANT4 simulation of the realized target. Left panel: Target vessel front view. On the right side the pipe and the laser entrance flange are drawn too. Right panel: inside of the cavity, the mirror is represented in cyan. The thin dark-gray slab is the lead absorber 
The laser light is injected in the cavity through a single $\mathrm{ZnS}$ optical window placed on the outer side of a pipe that is also the main support of the vessel inside the cryogenic cylinder.

The gas is injected from the rear of the pressurized vessel.

\subsubsection{Monte Carlo simulations of the target}

Dedicated Monte Carlo simulations have been developed for the data analysis's study of the systematics, the gas target assembly and the detector's system [50]. The same simulation code based on GEANT4 is being used for the final target layout optimization.

Panel g in Fig. 2 shows the energy spectra that have been obtained in the time slot chosen for laser injection after the beam arrival. In the 2016 target, the background comes mostly from muons transferred from $\mu^{-} \mathrm{p}$ to light elements, more specifically from bremsstrahlung photons generated by decay electrons.

This situation can change significantly with a much smaller gas target and with addition of the cavity mirrors and supports inside the vessel. A balance between competing physical processes must be found, taking into account also technical constraints especially related to the mirrors substrates and supports.

By means of simulations of the target system and considering the final gas target conditions, pressure of 7 bar at $80 \mathrm{~K}$, an analysis of the delayed background generated in the $\mathrm{LaBr}_{3}(\mathrm{Ce})$ detectors has been carried out to guide the final constructive design. In Fig. 10, a breakdown of the contribu-

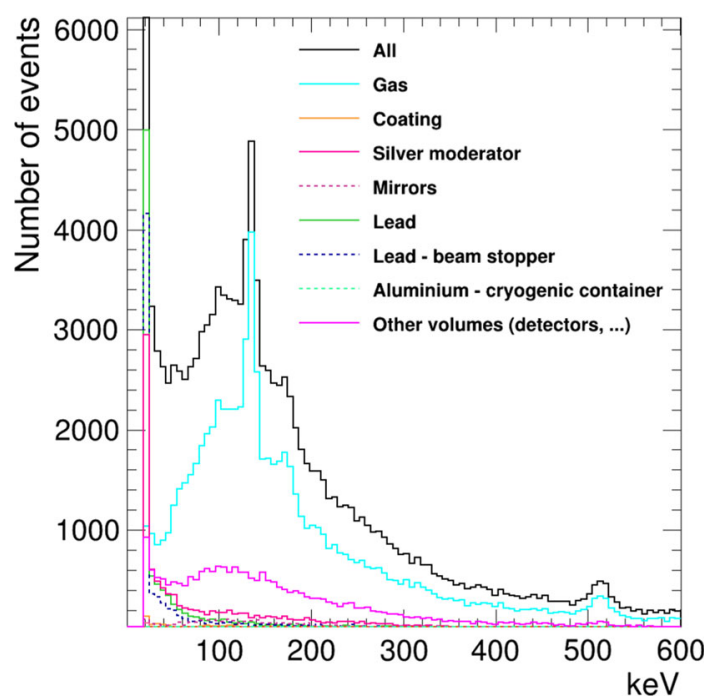

Fig. 10 Simulation of the delayed X-rays energy spectrum generated in the target volume. The signal contribution from target materials acting as a source has been singled out. All optical and mechanical elements have been considered. "Other volumes" is mainly due to the aluminium pressurized vessel, but includes detectors, holders and all other materials

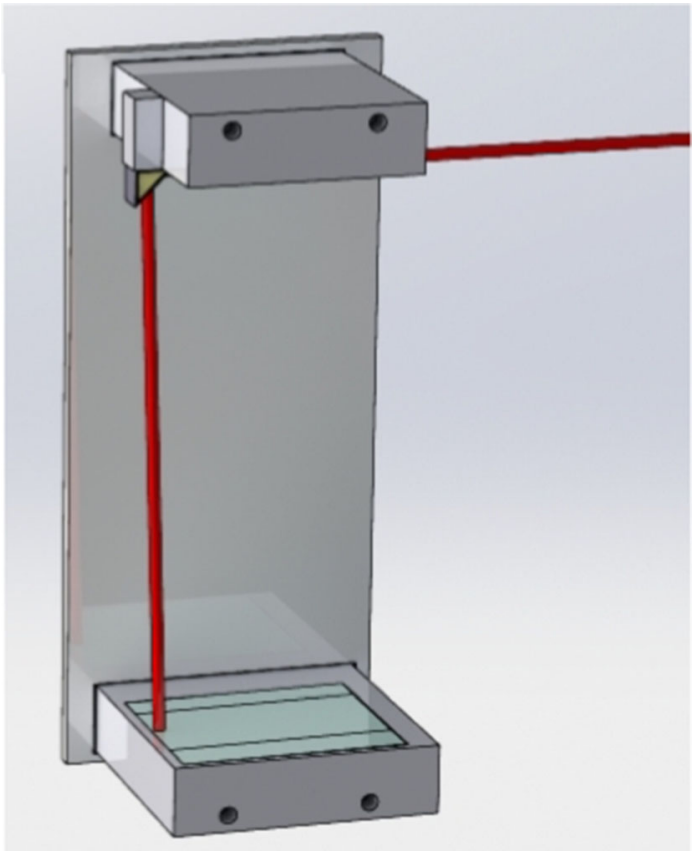

Fig. 11 Sketch of the transverse multipass optical cavity. The injection laser light path is shown in red

tion to the background from the different target's elements is shown as an example. The GEANT4 simulation was modified in order to include the physical process of the muon transfer from hydrogen to oxygen. The two peaks shown in Fig. 10 present the oxygen main $\mathrm{K}_{\alpha}$-line (about $130 \mathrm{keV}$ ) and the un-resolved $\mathrm{K}_{\beta}$ and $\mathrm{K}_{\gamma}$ lines (about $160 \mathrm{keV}$ ). In this configuration, the signal coming from the gas amounts to more than $50 \%$ of the total detected spectra. Small or negligible contributions come from the single elements of the target: aluminium pressurized vessel lead absorber, mirrors support, mirrors, inner cavity coating. This is a successful result of the optimisation process in the design. In total, of the 1000 muons per spill, 10 stop in the gas and are illuminated by the laser.

\subsection{Multipass optical cavity}

The cross-section of the hyperfine spin-flip transition in $\mu^{-} \mathrm{p}$ atoms, being a dipole magnetic transition, is four orders of magnitude smaller than the $2 s-2 p$ Lamb shift transition [6]. A multipass optical cavity to detect atomic muon transitions has been already realized at PSI [51], but the FAMU experimental requirements for the multipass optical cavity pose much stricter design conditions. In order to have a detectable signal, the design, including material, shape and size, has been optimized to enhance the interaction path between the $\mu^{-} \mathrm{p}$ atoms and photons of the laser pulse and increase the overall probability of spin-flip transition [52]. 
First of all, in order to increase the energy density, the illuminated surface on the mirrors is reduced to few square centimeters. Furthermore, to increase the interaction path, the light is injected into the cavity at a grazing angle from the side. Figure 11 shows a sketch of cavity. The laser is injected into the cavity and reflected almost parallel to the cavity axis by means of a small mirror placed near the top mirror.

A crucial point in the design of the cavity is the number of photon round trips, that is strictly correlated to the effective interaction length. Therefore, particular care has been posed in the design of the shape of the mirrors in order to obtain a long interaction path. The fused silica mirrors are coated with multilayer of $\mathrm{ZnS} / \mathrm{Ge}$ that provides a reflectivity $>99.98 \%$ at $6.78 \mu \mathrm{m}$. The cylindrical ends of the top mirror have a curvature radius of $54 \mathrm{~cm}$ and $17 \mathrm{~cm}$, while the curvature radius of the bottom mirror cylindrical ends are 15 and $42 \mathrm{~cm}$. The cylindrical ends are joined to the central plane piece by a system composed by a pair of screws and a thin sheet of invar that pushes the cylindrical ends on the flat part. Invar minimizes the thermal expansion at low temperature. A prototype is being build. Taking into account the substrate fabrication process, we expect the gap between the flat and the cylindrical parts to be about $15 \mu \mathrm{m}$.

The ray tracing is the fundamental simulation tool to design the cavity. MATLAB [53] was used for this purpose. Figure 12 reports on the ray-tracing and shows that the light fills almost completely the cavity volume. The light impinges on the mirrors about 1000 times. This effect has a direct consequence on the cavity photon life-time and on the equivalent interaction path of the light with the $\mu^{-} \mathrm{p}$, that are $304 \mathrm{~ns}$ and $91 \mathrm{~m}$, respectively. The ray path is quite sensible to the
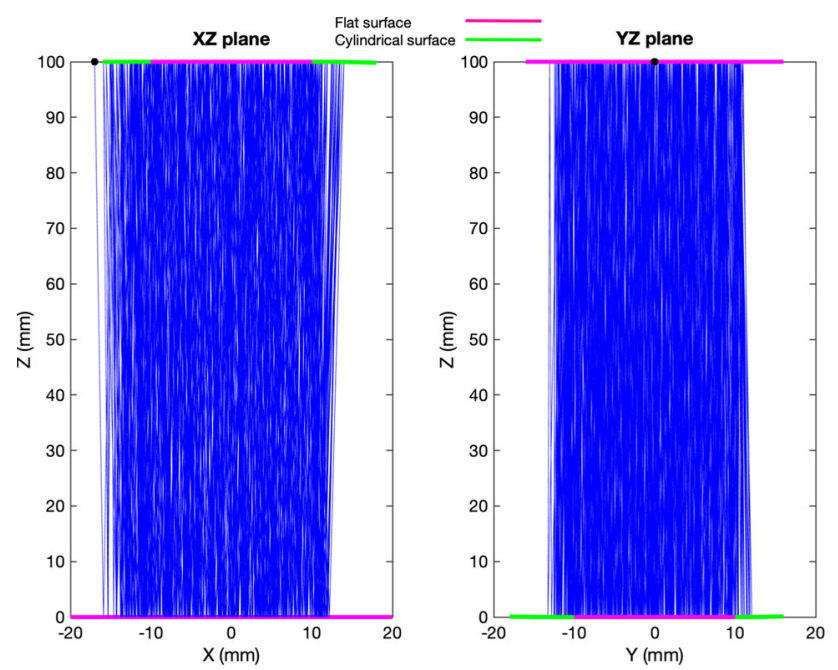

Fig. 12 Ray tracing simulation of the transverse optical cavity. The green line represents the cylindrical part of the mirror; the magenta line represents the flat part of mirror; the black dot represents the injection mirror

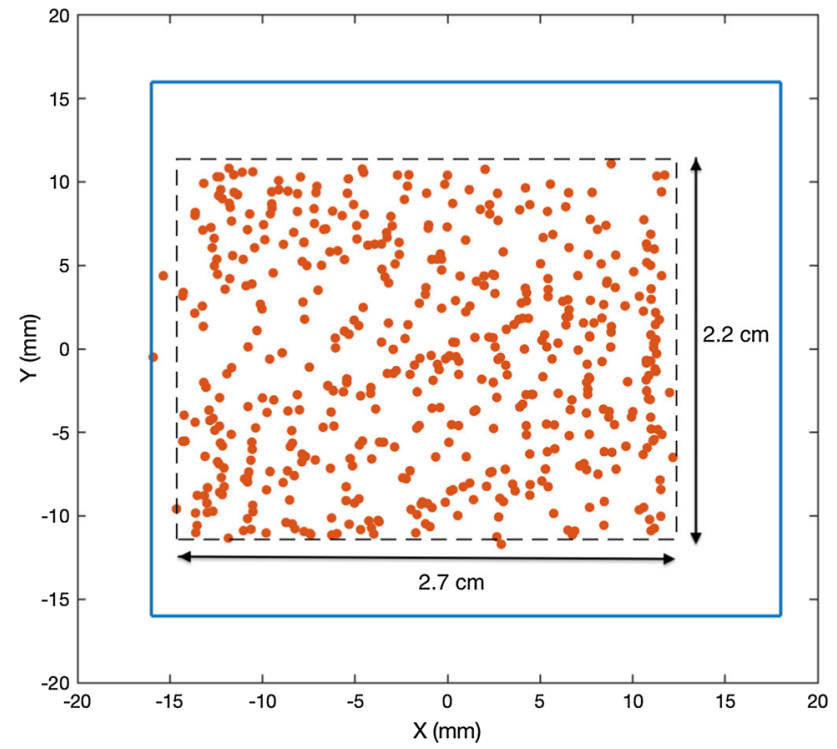

Fig. 13 Reflection spots on the top mirrors. The dashed line shows an estimation of illuminated surface

injection angle. Figure 13 shows the reflection spot maps on the top mirror; the estimate size of the illuminated surface (dashed line in Fig. 13) is about $2.7 \times 2.2 \mathrm{~cm}^{2}$. A preliminary study of the interference effect, taking into account the refocusing of the gaussian shape of the laser beam, has been performed and no significant perturbation of the energy density was found. The refocusing effect is negligible for the large curvature radius of the cylindrical part of the mirrors where, moreover, a small number of reflections occurs.

The light will be injected in the cavity with a couple of parabolic mirrors. This system will guarantee a stable alignment between the laser system and the multipass cavity. Moreover, with the parabolic mirrors system, it is possible to adjust the beam waist at the entrance of the cavity.

\subsection{Detectors system}

Muons stopping in the materials surrounding the gas will produce a large number of prompt muonic X-ray lines in the energy range of interest - from about $100 \mathrm{keV}$ to about $700 \mathrm{keV}$, including the oxygen lines, and among others, the typical calibration lines of the electron-positron annihilation at $511 \mathrm{keV}$, and the $662 \mathrm{keV}$ from cesium. The delayed Xray signal lines must be identified over a smooth $\mathrm{X}$-ray background that is mostly generated, via bremsstrahlung, by electrons coming from muon decay. This fact points to optimal beam control and a fast high efficiency X-ray detector system. The detection system that will be employed in the experiment have been thoroughly tested and improved in a progression of measurements campaigns between 2014 and 2018. The complete detection system will include: a beam profile mon- 
itor and an X-ray detection ensemble covering most of the solid angle.

\subsubsection{Beam profile monitor}

The presence of the beam profile monitor is dictated by the need to keep the beam parameters periodically under control and to avoid any unperceived drift in beam shape and position. The beam has to be centered on the gas target volume.

The beam monitor system realized during the FAMU preparatory phase has allowed us to: fine-tune the incoming beam, deliver timing information for DAQ readout and trigger, and monitor the intensity of each beam pulse. The system could rely on three different hodoscopes: two of $10 \times 10 \mathrm{~cm}^{2}$ active area and a smaller one of $3.2 \times 3.2 \mathrm{~cm}^{2}$ with $1 \mathrm{~mm}$ fibers $[54,55]$.

A new hodoscope has been designed to reduce the amount of material in front of the target and match the new target window. This hodoscope is based on $0.5 \times 0.5 \mathrm{~mm}^{2}$ square scintillating fibers read by $1 \times 1 \mathrm{~mm}^{2}$ Hamamatsu S12751050P SiPM. It will have $32+32 \mathrm{X}, \mathrm{Y}$ channels covering an active are of about $7.2 \times 7.2 \mathrm{~cm}^{2}$. The temperature gain drift of SiPM will be controlled by CAEN DT5485 voltage supplies. This hodoscope will be the only detector in front of the target: it will be placed between the beam collimator and the target cryostat.

\subsubsection{X-ray detectors}

To allow for a good signal to noise ratio and the extraction of the muonic X-ray lines with the best energy resolution and minimal events pile-up, a high light yield and fast decay time scintillating crystal is needed. From this point of view, $\mathrm{LaBr}_{3}(\mathrm{Ce})$ is the best crystal available today. In spectroscopy, the use of high purity germanium detectors (HPGe) exhibiting unbeatable energy resolution is valued as the best possible solution. However, HPGes are slow, work at liquid nitrogen temperatures, require voluminous cryostats and are very expensive. For those reasons FAMU will use a balanced mixture of both technologies already proven to be optimal solutions [56].

$\mathrm{LaBr}_{3}(\mathrm{Ce})$ offers the appropriate energy resolution, fast emission and excellent linearity, allowing also to maximize the detection efficiency while covering a large fraction of the solid angle. Light decay time is the shortest among inorganic scintillator materials (decay time at 97\% $16 \mathrm{~ns}$ ). The single channel coincidence resolving time is $0.45 \mathrm{~ns}$ for $2^{\prime \prime}$ $\mathrm{LaBr}_{3}(\mathrm{Ce})$ for the integrated detector crystals and photomultiplier element. The energy resolution $(7 \%$ at $140 \mathrm{keV}$ and $4 \%$ at $511 \mathrm{keV}$ ) is the best among scintillating crystals [57].

The system used for the 2015-18 data taking [58] is based on cylindrical $1^{\prime \prime} \mathrm{LaBr}_{3}(\mathrm{Ce})$ crystals arranged in a crown, read by photomultiplier tubes. Depending on the data tak- ing session we used 5-8 crystals in the crown. Up to now, data have been acquired and processed by $500 \mathrm{MHz}$ CAEN V1730 digitizers, in the framework of the general FAMU Data Acquisition System [59]. For the next run, the direct use of an on-board digital pulse processor with a speed up to $1 \mathrm{Gs} / \mathrm{s}$, performing noise reduction with an optimal (triangular) filter, is foreseen. $\mathrm{LaBr}_{3}(\mathrm{Ce})$ detectors read by photomultipliers will be arranged on a circular support that can contain up to 12 individual detectors, all facing the center of the target's optical cavity. The main module, shown in Fig. 14, is currently being assembled. Two additional circular arms may be added. This will allow a good coverage of the target volume improving, as far as possible, the X-rays statistic.

The collaboration is also developing a complementary read-out system for the $\mathrm{LaBr}_{3}(\mathrm{Ce})$ crystals based on SiPM. With respect to SiPM, the PMT readout offers the advantage of better rise times ( $\sim 10 \mathrm{~ns}$ as compared to $20 \mathrm{~ns}$ ). However, the use of smaller crystal, $1 / 2^{\prime \prime}$ instead of $1^{\prime \prime}$, combined with SiPM arrays, compensates for this. Therefore, eight cubic 1/2" $\mathrm{LaBr}_{3}(\mathrm{Ce})$ with $\mathrm{SiPM}$ array readout have been recently tested and were used to instrument the most inaccessible regions around the target $[60,61]$. The readout

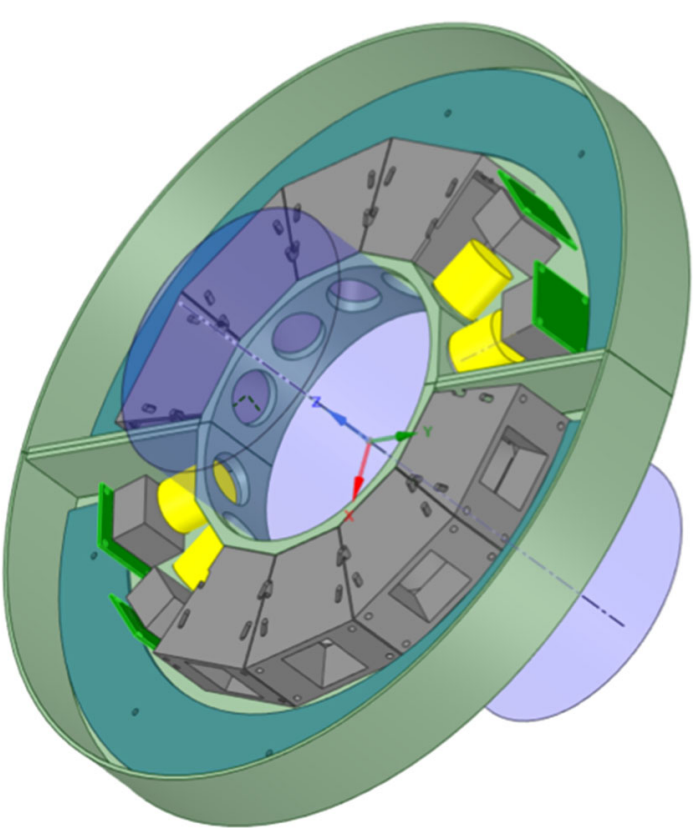

Fig. 14 CAD drawing of the main detection system. The cryogenic container of the gaseous target is shown as light blue cylinder. Each individual detector is composed of: the scintillating crystal $\mathrm{LaBr}_{3}(\mathrm{Ce})$ (in yellow) that is coupled with an Ultra High Quantum Efficiency photomultiplier (gray box) and the front-end electronics are housed on PCBs (green slabs). The detectors are housed in light-tight holders (dark gray) firmly attached to the circular aluminium support (in light green, with circular holes). The final assembly will differ from this drawing: one of detector module will not be mounted to make room to the laser injection system 
was based on a $4 \times 4$ array of $3 \times 3 \mathrm{~mm}^{2}$ Hamamatsu S13361 TSV SiPM. The output signals are summed up on a custom made PCB and digitized by CAEN V1730 digitizers. The signal is about $100-200 \mathrm{mV}$ for $622 \mathrm{keV}$, one of the lines used for calibration, and no amplification is needed in our range of interest. Sixteen of these $1 / 2^{\prime \prime} \mathrm{LaBr}_{3}(\mathrm{Ce})$ detectors will be placed around the target in front of the other ring of $\mathrm{LaBr}_{3}(\mathrm{Ce})$ detectors.

High purity germanium solid-state detectors have been equipped with a custom fast read-out electronics. In this way, HPGe detectors can be used as cross reference system complementing the $\mathrm{LaBr}_{3}(\mathrm{Ce})$ scintillation based detectors. Their readout is done through CAEN V1724 digitizers.

$\mathrm{LaBr}_{3}(\mathrm{Ce})$ scintillating crystals and HPGe detectors with dedicated fast electronics have already been tested in most realistic conditions on the RIKEN-RAL muon beam [58]. The ability to withstand the event flux originating from the muon beam having a double pulse structure, each with $70 \mathrm{~ns}$ FWHM width, $320 \mathrm{~ns}$ apart has been proven. The $\mathrm{LaBr}_{3}(\mathrm{Ce})$ detection system met the requirements, both on energy and time resolution, correctly reconstructing the relevant characteristic X-rays and the lifetimes of the muonic atoms [7,42]. HPGe detectors, although slower, have been used as a crosscheck measurement of the temperature dependence of the muon transfer rate to oxygen [39].

Figure 15 shows the arrangement of the detectors in the final setup: around the target are placed (1) a ring of 1/2" $\mathrm{LaBr}_{3}(\mathrm{Ce})$ read by SiPM; (2) a ring of $1^{\prime \prime} \mathrm{LaBr}_{3}(\mathrm{Ce})$ read by PMTs; and (3) one HPGe detector with its cooling tank. Another $1 / 2^{\prime \prime} \mathrm{LaBr}_{3}(\mathrm{Ce})$ detector's ring will possibly be placed behind the first two.

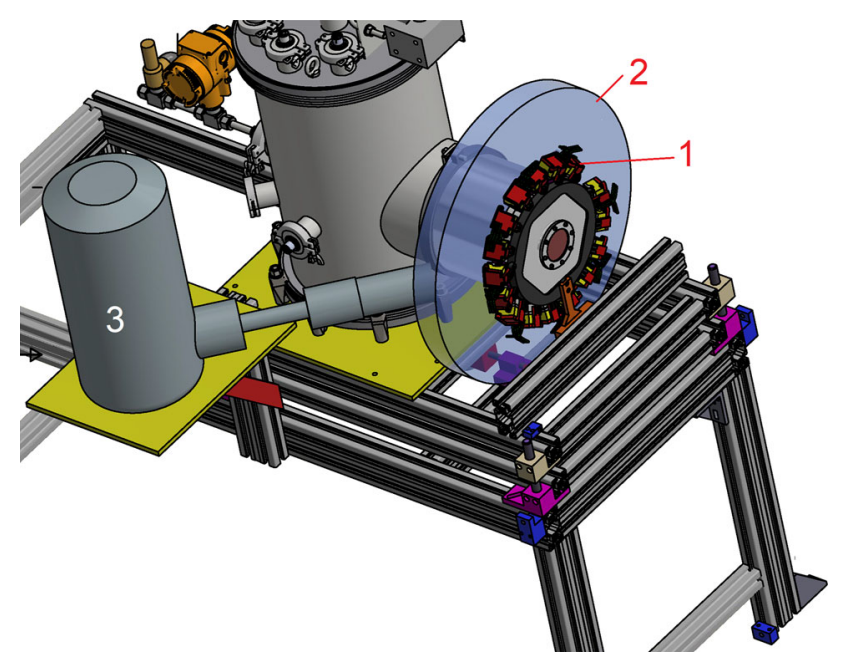

Fig. 15 CAD drawing of the detector positioning around the target

\section{Measurement planning}

\subsection{Rate evaluation from recent data}

In 2018, using the cryogenic target system prepared for the preliminary measurements, we have collected data with a hydrogen gas mixture with oxygen concentration of $1 \%$ at 7 bar and $80 \mathrm{~K}$. The beam momentum was set at $55 \mathrm{MeV} / \mathrm{c}$.

The useful time window is framed by the $\mu$ p's thermalization and the minimization of the beam induced noise and starts about $300 \mathrm{~ns}$ from the second beam pulse. Figure 16 reports the energy spectra for a selected time slot of $300 \mathrm{~ns}$ taken $300 \mathrm{~ns}$ after the second muon pulse arrives. In this time interval, the signal to noise ratio will be optimal as demonstrated by the simulation described in Sect. 2.3.2. About 44,000 delayed oxygen $\mathrm{K}$ lines photons were detected in 9 $h$ by five $\operatorname{LaBr}_{3}(\mathrm{Ce}) 1^{\prime \prime}$ detectors. From this, and using the results from simulations of the foreseen new target-cavity structure, we can predict the expected rates and hence the time needed for the spectroscopy measurement. From Monte Carlo simulations, we are able to evaluate the number of muons forming $\mu \mathrm{p}$ in the above named conditions assuming gas target-cavity dimensions of $2 \times 2 \times 10 \mathrm{~cm}^{3}$. With the final setup we expect that $8 \%$ of the muons form muonic hydrogen as compared with what we obtained in the 2018 run. We can rescale this value for a setup of 12 detectors. Assuming to have $24 \mathrm{~h}$ of stable laser condition, $12 \mathrm{~h}$ with and 12 $\mathrm{h}$ without laser, the number of expected signal is of about 12,000 in either situation. The statistical fluctuation of about $1 \%$ will be small enough to reach our goals, as illustrated in Sect. 2.2. With these statistics we can expect a signal to noise ratio ranging from 4 to 20 , depending on the available laser energy. Various aspects, like enlarging the number of detectors to cover the useful fraction of the solid angle or the

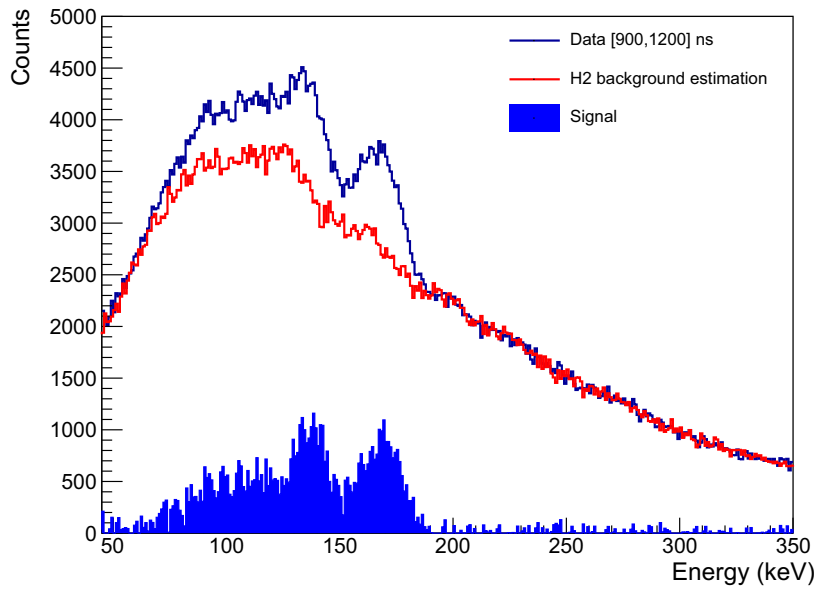

Fig. 16 Energy spectrum collected in $9 \mathrm{~h}$ during the 2018 data taking with five $\mathrm{LaBr}_{3}(\mathrm{Ce})$ detectors. The target was filled with $\mathrm{H}_{2} \quad 1 \% \mathrm{O}_{2}$ at $80 \mathrm{~K}, 7$ bars 
final X-ray detection efficiency, could lead to a redefinition of the time needed for each wavelength measurement.

\subsection{Number of measurements}

To evaluate the number of measurements, a wavelength window around the expected resonance position should be defined. Equation 1 expresses the resonance position, i.e. the hyperfine splitting energy, as a function of the Zemach radius. Published values of the Zemach radius [14-17,20,34], listed in Sect. 2.2, define an interval that ranges from 1.021 to $1.119 \mathrm{fm}$. This corresponds to an interval of width $0.00013 \mathrm{eV}$ in the hyperfine splitting energy or, equivalently, $0.00013 \mathrm{eV}$ $\times 0.2410^{6}(\mathrm{GHz} / \mathrm{eV})=30 \mathrm{GHz}$. Therefore, we have to scan an interval $\Gamma=30 \mathrm{GHz}$.

The number of needed wavelength steps may be estimated by dividing the interval $\Gamma$ by the FWHM of the laser linewidth $(\Delta v)$. The natural line Doppler broadening at $80 \mathrm{~K}$ is about $300 \mathrm{MHz}$. The laser linewidth should not exceed this value, a condition satisfied by the current FAMU laser. If we assume $\Delta v=0.3 \mathrm{GHz}$ that leads to 100 steps. If we proceed with frequency steps equal to the FWHM of the laser linewidth, it may happen that the resonance occurs inbetween two steps, at a frequency for which the intensity of the laser is only half of the maximum and the transition probability would be considerably reduced. Therefore, in the final measurement, we will need more measurements, namely between 100 and 200 .

\section{Conclusion}

In this paper we described the plan to measure the $h f s$ in the ground state of muonic hydrogen proposed by the FAMU collaboration, and have presented here, for the first time, the realisation of the complete experimental setup. In a progression of preparatory measurements, between 2014 and 2018, we have shown the feasibility of the proposed method-muon transfer to oxygen - and tested various aspect of the experiment. We plan to start a first session of data acquisition with a time span dedicated to assembly and testing of the complete experimental setup, including the laser. This first data acquisition run should last about 4 weeks, depending on the muon beam availability. This time span will allow us to gather about 20 points in frequency. The target will be filled with an oxygen and hydrogen mixture at constant temperature and pressure ( 7 bar, $80 \mathrm{~K}, 1.5 \%$ oxygen concentration). Each data-point measurement will require us to run for $24 \mathrm{~h}$, turning the laser on every other muon beam spill. This will allow us to minimize the systematic effects. This first run, with the full setup in the final configuration, is foreseen at the end of 2020. A second and longer run, with high-statistic data collection, will be held after the long shut down of the ISIS facility planned for 2020/2021.

Acknowledgements Authors would like to thank RIKEN-RAL and ISIS-STFC for the beam time and technical support during experiments. We are grateful of the constant support of the INFN CSN3 financing board. We thank Elettra for support on the laser work, and in particular, the SPIE-ICTP Anchor Research Program funded generously by the International Society for Optics and Photonics (SPIE). The research activity presented in this paper has been carried out with the partial support from the Bulgarian FNI under Grant DN08-17.

Data Availability Statement This manuscript has no associated data or the data will not be deposited. [Authors' comment: This paper presents the current setup of the FAMU experiment and the evaluation of the expected results, based on preliminary measurements and refined simulations. No new experimental data is presented here.]

Open Access This article is licensed under a Creative Commons Attribution 4.0 International License, which permits use, sharing, adaptation, distribution and reproduction in any medium or format, as long as you give appropriate credit to the original author(s) and the source, provide a link to the Creative Commons licence, and indicate if changes were made. The images or other third party material in this article are included in the article's Creative Commons licence, unless indicated otherwise in a credit line to the material. If material is not included in the article's Creative Commons licence and your intended use is not permitted by statutory regulation or exceeds the permitted use, you will need to obtain permission directly from the copyright holder. To view a copy of this licence, visit http://creativecomm ons.org/licenses/by/4.0/.

\section{References}

1. C.E. Carlson, V. Nazaryan, K. Griffioen, Phys. Rev. A 83, 042509 (2011)

2. S.G. Karshenboim, Phys. Rev. D 90, 053013 (2014)

3. S.G. Karshenboim, Phys. Rep. 422, 1-63 (2005)

4. S.G. Karshenboim, Phys. Rev. A 91, 012515 (2015)

5. D. Bakalov, E. Milotti, C. Rizzo, A. Vacchi, E. Zavattini, Phys. Lett. A 172, 277-280 (1993)

6. A. Adamczak, D. Bakalov, L. Stoychev, A. Vacchi, Nucl. Instrum. Methods B 281, 72-76 (2012)

7. A. Adamczak et al., J. Instrum. 11, P05007 (2016)

8. M. Weitz, A. Huber, F. Schmidt-Kaler, D. Leibfried, T.W. Hänsch, Phys. Rev. Lett. 72, 328-331 (1994)

9. H. Hellwig et al., IEEE Trans. Instrum. Meas. 19, 200-209 (1970)

10. L. Essen, R.W. Donaldson, M.J. Bangham, E.G. Hope, Nature 229, 110-111 (1971)

11. R. Pohl, R. Gilman, G.A. Miller, K. Pachucki, Annu. Rev. Nucl. Part. Sci. 63, 175-204 (2013)

12. A.M. Eides, H. Grotch, V.A. Shelyuto, Phys. Rep. 342, 63-261 (2001)

13. R.N. Faustov, A.P. Martynenko, G.A. Martynenko, V.V. Sorokin, Phys. Lett. B 733, 354-358 (2014)

14. A. Dupays, A. Beswick, B. Lepetit, C. Rizzo, D. Bakalov, Phys. Rev. A 68, 052503 (2003)

15. A.V. Volotka, V.M. Shabaev, G. Plunien, G. Soff, Eur. Phys. J. D 33, 23-27 (2005)

16. S.J. Brodsky et al., Phys. Rev. Lett. 94022001 (2005) [Phys. Rev. Lett. 94169902 (2005)]

17. J.L. Friar, I. Sick, Phys. Lett. 579B, 285 (2004)

18. R. Pohl et al., Science 353, 669-673 (2016) 
19. R. Pohl et al., Nature 466, 213-216 (2010)

20. A. Antognini et al., Science 339, 417-420 (2013)

21. A. Antognini, International Conference on Laser Spectroscopy, ICOLS 2015, Singapore. (2015). arXiv:1512.01765

22. M. Sato et al., Proceedings of the 20th Particles and Nuclei International Conference, Hamburg, 2014. https://doi.org/10.3204/ DESY-PROC-2014-04/67

23. A. Bertin et al., Phys. Lett. B 78, 355-359 (1978)

24. W. Ruckstuhl et al., Nucl. Phys. A 433, 634-648 (1985)

25. I. Beltrami et al., Nucl. Phys. A 451, 679-700 (1986)

26. L. Bracci, A. Vacchi, E. Zavattini, Z. Phys. C 56, S74-S79 (1992)

27. D. Guffanti, FAMU Collaboration, J. Phys. Conf. Ser. 689, 012018 (2016)

28. D. Bakalov, A. Adamczak, M. Stoilov, A. Vacchi, Phys. Lett. A 379, 151-156 (2015)

29. A. Adamczak et al., Atom. Data Nucl. Data Tables 62, 255-344 (1996)

30. A. Werthmüller et al., Hyperfine Interact. 116, 1-16 (1998)

31. A.T. Le, C.D. Lin, Phys. Rev. A 71, 022507 (2005)

32. S.G. Karshenboim, Phys. Lett. A 225, 97-106 (1997)

33. E.V. Cherednikova, R.N. Faustov, A.P. Martynenko, Nucl. Phys. A 703, 365-377 (2002)

34. J.C. Bernauer et al., Phys. Rev. C 90, 015206 (2014)

35. T. Matsuzaki et al., Nucl. Instrum. Methods A 465, 365-383 (2001)

36. A. Vacchi, E. Mocchiutti, D. Guffanti, F.A.M.U. Collaboration. RIKEN Accel Prog Rep 49, 230 (2016)

37. A. Vacchi, K. Ishida, E. Mocchiutti, N. Rossi, On Behalf of the FAMU Collaboration. RIKEN Accel. Prog. Rep. 50, 24 (2017)

38. E. Mocchiutti et al., Phys. Lett. A 384, 126667 (2020)

39. E. Furlanetto, Master thesis, Università degli studi di Trieste. (2016). http://www.infn.it/thesis/PDF/getfile.php? filename $=11690$-Furlanetto- magistrale.pdf

40. D. Guffanti, Master thesis, Università degli studi di Trieste. (2013). http://www.infn.it/thesis/PDF/getfile.php? filename $=9842-$ Guffanti- magistrale.pdf

41. L.P. Rignanese, Ph.D. thesis, Università di Bologna. (2019). http://www.infn.it/thesis/PDF/getfile.php? filename=13211-Rignanese- dottorato.pdf

42. E. Mocchiutti et al., J. Phys. Conf. Ser. 1138 (2018). arXiv: 170803172
43. D. Bakalov, A. Adamczak, M. Stoilov, A. Vacchi, Hyperfine Interact. 233, 97-101 (2015)

44. D. Bakalov, A. Beswick, A. Dupays, C. Rizzo, Can. J. Phys. 83, 351-356 (2005)

45. L.I. Stoychev et al., Laser Sources Appl. II Proc. 9135, 91350J (2014)

46. L.I. Stoychev et al., 2015 Fotonica AEIT Italian Conference Photonics Technology, Turin Italy, 6-8 May (2015)

47. L.I. Stoychev, H. Cabrera et al., DFG based high energy MID-IR tunable source with $30 \mathrm{pm}$ linewidth. Opt. Lett. (submitted)

48. L.I. Stoychev et al., Laser Phys. 29, 065801 (2019)

49. High-resolution transmission molecular absorption database. https://hitran.org and http://hitran.iao.ru/. Accessed Dec 2019

50. P. Danev et al., JINST 11, P03019 (2016)

51. J. Vogelsang et al., Opt. Express 22, 13050-13062 (2014)

52. D. Bakalov, M. Stoilov, EPJ Web Conf. 181, 01033 (2018). https:// doi.org/10.1051/epjconf/201818101033

53. MATLAB, 9.7.0.1190202 (R2019b) (The MathWorks Inc., Natick, Massachusetts, 2018)

54. M. Bonesini et al., JINST 12, C03035 (2017)

55. M. Bonesini et al., Nucl. Instrum. Methods A 936, 592 (2019)

56. A. Adamczack et al., JINST 13(12), P12033 (2018)

57. https://www.crystals.saint-gobain.com/products/ crystal-scintillation-materials

58. G. Baldazzi et al., J. Instrum. 12, C03067 (2017)

59. M. Soldani et al., Nucl. Instrum. Methods A 936, 327-328 (2019)

60. M. Bonesini et al., Proceedings of Science EPS-HEP2017, 777 (2017)

61. R. Benocci et al., Nucl. Instrum. Methods. A 936, (2019) 